\title{
Risk of de novo Hepatocellular Carcinoma after HCV Treatment with Direct-Acting Antivirals
}

\author{
Fabian Finkelmeier $^{a} \quad$ Georg Dultz $^{a} \quad$ Kai-Henrik Peiffer ${ }^{a}$ \\ Bernd Kronenberger $^{\mathrm{a}, \mathrm{b}} \quad$ Franziska Krauss $^{\mathrm{a}}$ Stefan Zeuzem ${ }^{\mathrm{a}}$ \\ Christoph Sarrazin $^{a, c}$ Johannes Vermehren ${ }^{a}$ Oliver Waidmann ${ }^{a}$ \\ a Schwerpunkt Gastroenterologie und Hepatologie, Medizinische Klinik 1, \\ Universitätsklinikum Frankfurt, Frankfurt am Main, Germany; ${ }^{b}$ Gastroenterologie, \\ Hepatologie, Allgemeine Innere Medizin, Herz-Jesu-Krankenhaus Fulda, Fulda, Germany; \\ 'Schwerpunkt Gastroenterologie und Hepatologie, Medizinische Klinik 2, \\ St. Josefs-Hospital Wiesbaden, Wiesbaden, Germany
}

\section{Keywords}

Hepatitis C · Hepatocellular carcinoma - Direct-acting antivirals

\begin{abstract}
Background and Aims: The aim of the study was to evaluate the risk of hepatocellular carcinoma (HCC) development after treatment with direct-acting antivirals (DAAs) and to compare $\mathrm{HCC}$ occurrence in these patients with that among patients treated with interferon (IFN)based therapies. Methods: We analyzed a large cohort with chronic hepatitis $C$ virus patients for the onset of new HCC after DAA treatment. A historical IFN-treated cohort was investigated for comparison. Results: A total of 819 patients were included in the DAA group. The median follow-up period was 263 days $(0-1,001)$. Twenty-five patients $(3.6 \mathrm{HCCs} / 100$ personyears; $3.1 \%$ ) were diagnosed with de novo HCC within the time of observation. No patient without cirrhosis had developed HCC. Patients with newly diagnosed HCC were mostly male, older, and treatment-experienced and had a lower 12-week sustained virologic response (SVR12) rate and higher levels of liver inflammation markers. The median time to HCC was 312 days $(0-880)$. Investigation of the subcohort of 269 cirrhotic patients yielded an HCC rate of $8.9 \mathrm{HCCs} / 100$ person-years. In this cohort, non-SVR12 was an independent risk factor for de novo HCC (HR 4.48; 95\% CI 1.51-13.12; $p=0.007)$. Twenty-four patients (96\%) with new HCC
\end{abstract}

J.V. and O.W. contributed equally to this work. 
were Child-Pugh class A and 17 (68\%) were diagnosed in early BCLC stage A. For the IFNtreated patients, we calculated an overall risk of HCC occurrence of $1.3 / 100$ person-years (19 patients out of $351 ; 5.4 \%)$. The median time to diagnosis was 38.8 months ( $0-113)$. Conclusion: The de novo HCC rates did not differ between the DAA-treated patients and those who received IFN. Achievement of SVR is of utmost importance for HCC prevention.

(C) 2018 S. Karger AG, Basel

\section{Introduction}

Chronic infection with the hepatitis $\mathrm{C}$ virus (HCV) is a major health burden and one of the main causes of chronic liver disease, with an estimated $2-3 \%$ of the world population being chronically infected $[1,2]$. Chronic HCV infection progresses to liver cirrhosis via an inflammatory process in $20-30 \%$ of all patients $[3,4]$. However, the progression rates show high variability [5].

Hepatocellular carcinoma (HCC) is the most common malignant primary liver cancer and the third leading cause of cancer-related death, affecting more than half a million patients annually [6]. Cirrhosis is the predominant risk factor for HCC development. The cumulative risk for HCC development among patients with cirrhosis ranges from 5 to $30 \%$ within 5 years $[6,7]$, while the rate of patients with chronic HCV infection and established cirrhosis developing HCC is around 3.5\% per year [8-10]. Patients with established fibrosis are at higher risk of developing HCC than patients in a lower fibrosis stage $[11,12]$.

With the introduction of the first NS5B polymerase inhibitor sofosbuvir (SOF) in 2014, interferon (IFN)-free treatment became available, and a sustained virologic response (SVR) could be achieved in $>90 \%$ of patients [13]. While patients with cirrhosis are often not eligible to undergo IFN-based treatment, direct-acting antivirals (DAAs) including NS5B and NS5A inhibitors are safe and effective for patients with advanced liver disease, including those with decompensated cirrhosis [14]. The high SVR rates leading to stabilization and improvement of liver function raise hope that treatment with DAAs can reduce the rate of HCC among patients with former chronic HCV infection. However, recently a discussion has started about a possible link between DAA treatment and a higher risk of HCC occurrence and recurrence $[15,16]$.

The aim of this study was to investigate the incidence of newly diagnosed HCC in a cohort of patients with chronic HCV infection treated with DAAs without any history of HCC. Furthermore, we compared our results with those for a historical cohort of patients treated with IFN-based regimens.

\section{Patients and Methods}

\section{Selection of Patients}

DAA Cohort

Between January 2014 (approval of SOF) and August 2016, patients with confirmed chronic HCV infection undergoing treatment with DAAs at the hepatology outpatient clinic of the University Hospital Frankfurt were consecutively and prospectively enrolled as part of a multicenter resistance database study. The cohort was retrospectively analyzed for HCC development. The study was performed in accordance with the 1975 Declaration of Helsinki. The Ethics Committee of the University Hospital Frankfurt approved the study.

For the present analysis, the inclusion criteria comprised chronic HCV infection and treatment with DAAs. The exclusion criteria were an age below 18 years, a history of HCC or de novo HCC before the start of DAA treatment, or coinfection with HIV. All patients underwent HCC screening within 4 weeks before the start of treatment by abdominal ultrasound or dynamic imaging including computed tomography (CT) 


\section{Liver Cancer}

\begin{tabular}{l|l}
\hline Liver Cancer \\
\hline DOI: 10.1159/000486812 & $\begin{array}{l}\text { @ 2018 S. Karger AG, Basel } \\
\text { www.karger.com/lic }\end{array}$ \\
\hline
\end{tabular}

Finkelmeier et al.: HCC Risk following HCV Treatment

or magnetic resonance imaging (MRI). Cirrhosis was diagnosed if typical clinical features, endoscopic findings, laboratory results, imaging results, or results of liver biopsy or transient elastography were present.

Follow-up time was defined as the time from the start of treatment with DAAs until the last visit with imaging of the liver including ultrasound, CT, or MRI, a renewed diagnosis of HCC, or death.

IFN-Treated Historical Comparison Cohort

For comparison, we included a historical cohort of patients with chronic HCV infection with and without cirrhosis treated with IFN-based regimens. Between 1998 and 2010, patients with confirmed chronic HCV infection with and without cirrhosis presenting to the hepatology outpatient clinic of the University Hospital Frankfurt for treatment with IFN were retrospectively identified in the clinic's patient database. The Ethics Committee of the University Hospital Frankfurt approved the analysis.

The inclusion criteria comprised chronic HCV infection and treatment with IFN/peginterferon \pm ribavirin (RBV). The exclusion criteria were an age below 18 years, a history of HCC or de novo HCC before the start of treatment, or coinfection with HIV. The primary endpoints of follow-up were development of cirrhosis, development of HCC, or death. All patients were followed up from the start of therapy until one of the defined endpoints. Successful antiviral therapy was defined as undetectable HCV RNA 24 weeks after the end of therapy (SVR24).

\section{Assessment of Baseline and Treatment-Related Patient Parameters}

The electronic hospital charts were retrospectively analyzed for baseline demographic data and laboratory results. All patients were analyzed for SVR12 (IFN cohort for SVR24; undetectable HCV RNA in the blood of patients 12 or 24 weeks after the end of antiviral therapy).

All patients were retrospectively analyzed for imaging of the liver; the latest image was used as a cutoff. If HCC was suspected, all results from further imaging and diagnostic procedures were evaluated. HCC was diagnosed according to current guidelines by dynamic imaging techniques with 4-phase multidetector CT scanning or dynamic contrast-enhanced MRI and the typical hallmark of HCC (hypervascularity in the arterial phase with washout in the portal venous or delayed phases) or by histopathological examination of biopsies [17]. Fibrosis was assessed by transient elastography results before the start of treatment if available. The cutoff values were chosen as follows: $7.1 \mathrm{kPa}$ for fibrosis stage $(\mathrm{F})>2,9.5 \mathrm{kPa}$ for F >3, and $12.5 \mathrm{kPa}$ for F4, as described previously $[18,19]$.

Treatment of HCV with DAAs

The patients received one of the following regimens: (1) SOF (nucleoside NS5B polymerase inhibitor) and ledipasvir (NS5A inhibitor) \pm RBV for genotypes 1 and 4-6; (2) SOF and daclatasvir (NS5A inhibitor) \pm RBV for genotypes 1 and 3; (3) paritaprevir/ritonavir (ritonavir-boosted protease inhibitor), ombitasvir (NS5A inhibitor), and dasabuvir (nonnucleoside NS5B polymerase inhibitor) \pm RBV for genotype 1; (4) SOF and simeprevir (NS3 protease inhibitor) \pm RBV for genotype 1; and (5) SOF + RBV for genotypes 2 and 3. RBV was administered based on the current guideline recommendations [20,21].

Virologic response to therapy was assessed by quantitative HCV RNA assessment using real-time PCR with a limit of detection of $15 \mathrm{IU} / \mathrm{mL}$ at week 12 of follow-up (SVR12).

\section{Treatment of HCV in the IFN Cohort}

The patients received IFN/peginterferon alfa-2a or -2b plus RBV for 24 weeks (genotypes 2 and 3 ) or 48 weeks (all other genotypes). Peginterferon alfa-2a was administered subcutaneously at a dose of $180 \mu \mathrm{g}$ per week. The doses of peginterferon alfa- $2 \mathrm{~b}$ and RBV were weight based according to the respective recommendations of the manufacturers.

\section{Statistical Analysis}

This trial was designed as a retrospective cohort study. HCV patients were prospectively included from the start of DAA treatment at the outpatient department. IFN-treated patients were retrospectively collected from the patient database of the clinic. All patients were followed up until death or last contact. The primary endpoint was development of HCC. Continuous variables are shown as medians and full ranges, and categorical variables are reported as frequencies and percentages. 
Finkelmeier et al.: HCC Risk following HCV Treatment

Fig. 1. Flowchart of included and excluded patients treated with direct-acting antivirals (DAAs) and numbers of patients with hepatocellular carcinoma (HCC). SVR, sustained virologic response.

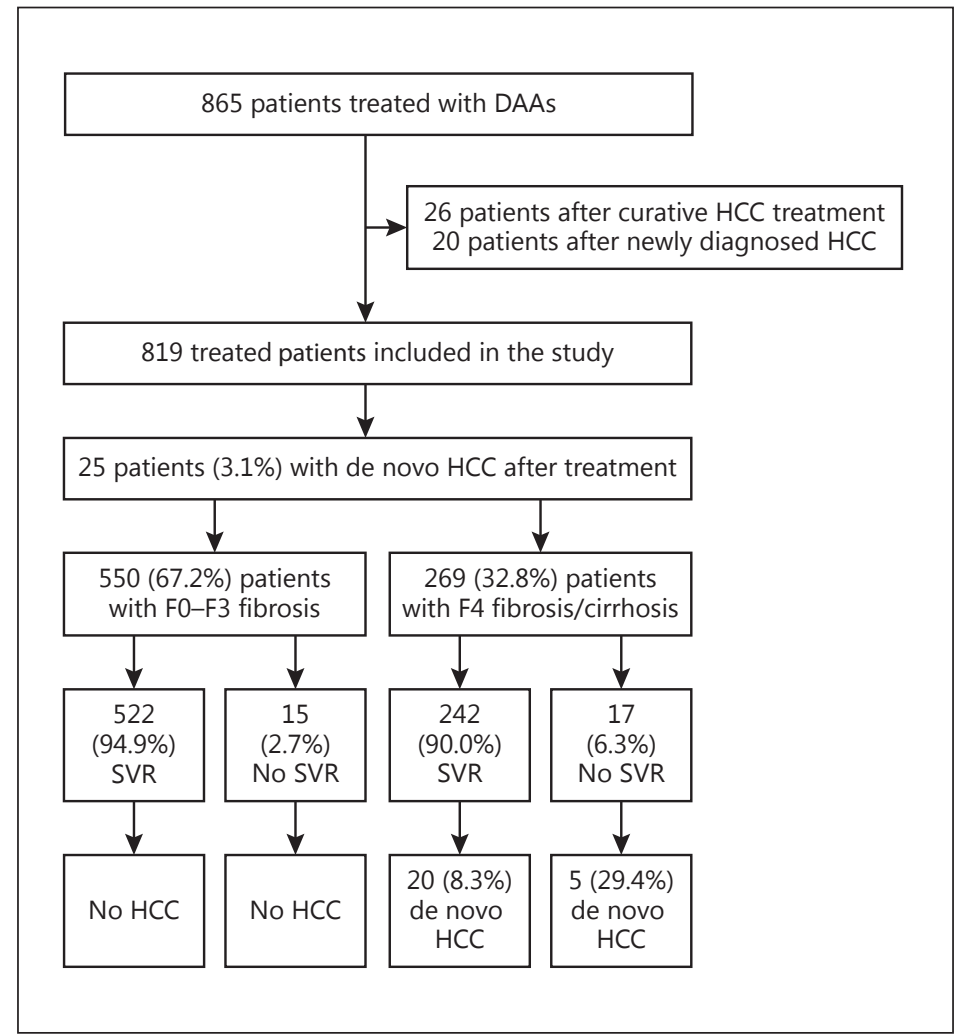

Differences between the two patient cohorts were determined using the nonparametric WilcoxonMann-Whitney and Kruskal-Wallis tests or the Fisher exact test. For subanalysis of multiple comparisons, Bonferroni correction was used. $p$ values $<0.05$ were considered to be significant.

Predictors of HCC were determined using a univariate Cox proportional-hazards regression model. HCC was recorded as an event. For the assessment of independent predictors of HCC, a multivariate Cox proportional-hazards regression model with forward stepwise (likelihood ratio) entry was used. Survival curves with the estimated hazard ratios were calculated with the Cox regression model. The statistical analyses were performed with SPSS (version 22.0; IBM, New York, NY, USA) and GraphPad Prism 5.0a (GraphPad Software, La Jolla, CA, USA).

\section{Results}

A total of 865 patients were treated with DAAs in the defined time period. Twenty-six of these 865 patients were excluded because they had undergone curative HCC treatment before the start of treatment, and 20 patients were excluded because DAA therapy was started after diagnosis of de novo HCC. Thus, a total of 819 patients were included in the final analysis (Fig. 1).

The median follow-up after the start of DAA treatment was 263 days, ranging from 0 to 1,001 days; 269 (32.8\%) had established cirrhosis, mostly Child-Pugh class A (211 patients, $25.8 \%$ ). According to the results of liver elastography, 322 (39.3\%) had no fibrosis, while 269 (32.8\%) had F1-3 fibrosis.

Twenty-five patients ( $3.1 \%$ or $3.6 \mathrm{HCCs} / 100$ person-years) were diagnosed with de novo HCC in the entire DAA cohort within the time of observation. Among the patients with cirrhosis, the HCC rate was 8.9 HCCs/100 person-years. All 25 patients with de novo HCC had cirrhosis. 


\section{Liver Cancer}

\begin{tabular}{l|l}
\hline Liver Cancer \\
\hline DOI: 10.1159/000486812 & $\begin{array}{l}\text { C) 2018 S. Karger AG, Basel } \\
\text { www.karger.com/lic }\end{array}$ \\
\hline
\end{tabular}

Finkelmeier et al.: HCC Risk following HCV Treatment

Table 1. Patient characteristics of the DAA-treated patients

\begin{tabular}{|c|c|c|c|c|}
\hline Parameter & All patients & $\begin{array}{l}\text { No } \\
\text { diagnosed HCC }\end{array}$ & $\begin{array}{l}\text { Newly } \\
\text { diagnosed HCC }\end{array}$ & $p$ value \\
\hline \multicolumn{5}{|l|}{ Epidemiology } \\
\hline Patients, $n(\%)$ & 819 & 794 (96.9) & $25(3.1)$ & NA \\
\hline Gender $(\mathrm{m} / \mathrm{f}), n(\%)$ & $470(57.4) / 349(42.6)$ & $448(56.4) / 346(43.6)$ & $22(88.0) / 3(12.0)$ & 0.002 \\
\hline Age, years & $55(18-86)$ & $55(18-86)$ & $60(45-78)$ & 0.002 \\
\hline Follow-up, days & $263(0-1,001)$ & $261(0-1,001)$ & $312(0-880)$ & 0.236 \\
\hline De novo HCC, $n(\%)$ & $25(3.1)$ & $0(0.0)$ & $25(100.0)$ & NA \\
\hline Cirrhosis, $n(\%)$ & $269(32.8)$ & $244(30.7)$ & $25(100.0)$ & $<0.001$ \\
\hline Child-Pugh class, $n(\%)$ & & & & 0.088 \\
\hline A & $211(25.8)$ & $187(23.6)$ & $24(96.0)$ & \\
\hline $\mathrm{B}$ & $50(6.1)$ & $49(6.2)$ & $1(4.0)$ & \\
\hline $\mathrm{C}$ & $8(1.0)$ & $8(1.0)$ & $0(0.0)$ & \\
\hline MELD score & $9(6-24)$ & $9(6-24)$ & $9(6-13)$ & 0.967 \\
\hline Fibrosis stage, $n(\%)$ & & & NA & NA \\
\hline F0 & $322(39.3)$ & $322(40.6)$ & & \\
\hline $\mathrm{F} 1$ & $90(11.0)$ & $90(11.3)$ & & \\
\hline $\mathrm{F} 2$ & $68(8.3)$ & $68(8.6)$ & & \\
\hline F3 & $70(8.5)$ & $70(8.8)$ & & \\
\hline HBsAg positive, $n(\%)$ & $8(1.0)$ & $8(1.0)$ & $0(0.0)$ & 1.0 \\
\hline Liver stiffness, $\mathrm{kPa}$ & $5.6 \pm 9.4$ & $5.4 \pm 9.2$ & $23.0 \pm 24.4$ & $<0.001$ \\
\hline \multicolumn{5}{|l|}{ Genotype, $n(\%)$} \\
\hline 1 & $605(73.9)$ & $587(73.9)$ & $18(72.0)$ & \\
\hline 2 & $50(6.1)$ & $50(6.3)$ & $0(0.0)$ & \\
\hline 3 & $125(15.3)$ & $120(15.1)$ & $5(20.0)$ & \\
\hline 4 & $39(4.8)$ & $37(4.7)$ & $2(8.0)$ & \\
\hline Previous therapies, $n(\%)$ & & & & 0.001 \\
\hline Naive & $445(54.3)$ & $440(55.4)$ & $5(20.0)$ & \\
\hline Experienced & $374(45.7)$ & $354(44.6)$ & $20(80.0)$ & \\
\hline Treatment regime, $n(\%)$ & & & & 0.003 \\
\hline $3 \mathrm{D}$ & $120(14.7)$ & $117(14.7)$ & $3(12.0)$ & \\
\hline $\mathrm{SOF}+\mathrm{DCV} \pm \mathrm{RBV}$ & $151(18.4)$ & $145(18.3)$ & $6(24.0)$ & \\
\hline $\mathrm{SOF}+\mathrm{LDV} \pm \mathrm{RBV}$ & $384(46.9)$ & $375(47.2)$ & $9(36.0)$ & \\
\hline $\mathrm{SOF}+\mathrm{RBV}$ & $104(12.7)$ & $102(12.8)$ & $2(8.0)$ & \\
\hline $\mathrm{SOF}+\mathrm{SMV} \pm \mathrm{RBV}$ & $54(6.6)$ & $52(6.5)$ & $2(8.0)$ & \\
\hline Others & $6(0.7)$ & $3(0.4)$ & $3(12.0)$ & \\
\hline SVR12, $n(\%)$ & & & & 0.003 \\
\hline Yes & $764(93.3)$ & $744(93.7)$ & $20(80.0)$ & \\
\hline No & $32(3.9)$ & $27(3.4)$ & $5(20.0)$ & \\
\hline Lost to follow-up & $23(2.8)$ & $23(2.9)$ & $0(0.0)$ & \\
\hline Platelets/nL & $182(5-2,116)$ & $186(5-2,116)$ & $111(18-164)$ & $<0.001$ \\
\hline Bilirubin, mg/dL & $0.6(0.1-9.5)$ & $0.6(0.1-9.5)$ & $1.05(0.5-2.7)$ & $<0.001$ \\
\hline Albumin, mg/dL & $4.3(2.0-5.3)$ & $4.4(2.0-5.3)$ & $3.9(2.7-4.6)$ & $<0.001$ \\
\hline Creatinine, $\mathrm{mg} / \mathrm{dL}$ & $0.8(0.43-10.72)$ & $0.8(0.43-10.72)$ & $0.82(0.54-1.58)$ & 0.389 \\
\hline Prothrombin time, $\%$ & $99(22.0-143.0)$ & $99(22.0-143.0)$ & $84(58.0-110.0)$ & 0.001 \\
\hline GGT, U/L & $71(9-1,510)$ & $68(9-1,510)$ & $180(37-916)$ & $<0.001$ \\
\hline GOT, U/L & $57(11-1,243)$ & $56(11-502)$ & $88(31-268)$ & 0.014 \\
\hline GPT, U/L & $67(12-1,243)$ & $67(12-1,243)$ & $73(30-195)$ & 0.655 \\
\hline
\end{tabular}

Variables are expressed as medians (full ranges), numbers (proportions), or median $\pm \mathrm{SD}$, as appropriate. All $p$ values reported are two-sided. Statistical significance (in bold typeface) was defined as $p \leq 0.05$. DAA, direct-acting antiviral; HCC, hepatocellular carcinoma; MELD, Model for End-Stage Liver Disease; HBsAg, hepatitis B surface antigen; SOF, sofosbuvir; DCV, daclatasvir; RBV, ribavirin; LDV, ledipasvir; SMV, simeprevir; SVR12, sustained virologic response, undetectable HCV RNA 12 weeks after the end of therapy. 


\section{Liver Cancer}

\begin{tabular}{l|l}
\hline Liver Cancer \\
\hline DOI: 10.1159/000486812 & $\begin{array}{l}\text { (c) 2018 S. Karger AG, Basel } \\
\text { www.karger.com/lic }\end{array}$ \\
\hline
\end{tabular}

Finkelmeier et al.: HCC Risk following HCV Treatment

Table 2. Patient characteristics of the DAA-treated patients with cirrhosis

\begin{tabular}{|c|c|c|c|c|}
\hline Parameter & $\begin{array}{l}\text { All patients } \\
\text { with cirrhosis }\end{array}$ & $\begin{array}{l}\text { No } \\
\text { diagnosed HCC }\end{array}$ & $\begin{array}{l}\text { Newly } \\
\text { diagnosed HCC }\end{array}$ & $p$ value \\
\hline \multicolumn{5}{|l|}{ Epidemiology } \\
\hline Patients, $n(\%)$ & 269 & $244(90.7)$ & $25(9.3)$ & \\
\hline Gender (m/f), $n(\%)$ & $183(68.0) / 86(32.0)$ & $161(66.0) / 83(34.0)$ & $22(88.0) / 3(12.0)$ & 0.025 \\
\hline Age, years & $58(29-86)$ & $58(29-86)$ & $60(45-78)$ & 0.112 \\
\hline Follow-up, days & $364(0-950)$ & $364(0-950)$ & $312(0-880)$ & 0.856 \\
\hline De novo HCC, $n(\%)$ & $25(9.3)$ & - & $25(100.0)$ & \\
\hline Child-Pugh class, $n(\%)$ & & & & 0.088 \\
\hline A & $211(78,4)$ & $187(76.6)$ & $24(96.0)$ & \\
\hline $\mathrm{B}$ & $50(18.6)$ & $49(20.1)$ & $1(4.0)$ & \\
\hline $\mathrm{C}$ & $8(3.0)$ & $8(3.3)$ & $0(0.0)$ & \\
\hline MELD score & $9(6-24)$ & $9(6-24)$ & $9(6-13)$ & 0.872 \\
\hline Liver stiffness, $\mathrm{kPa}$ & $20.6(6.1-63.9)$ & $20.3(6.2-63.9)$ & $23(6.1-48.0)$ & 0.631 \\
\hline \multicolumn{5}{|l|}{ Genotype, $n(\%)$} \\
\hline 1 & $187(69.5)$ & $169(96.3)$ & $18(72.0)$ & \\
\hline 2 & $12(4.5)$ & $12(4.9)$ & $0(0.0)$ & \\
\hline 3 & $54(20.1)$ & $49(20.1)$ & $5(20.0)$ & \\
\hline 4 & $16(5.9)$ & $14(5.7)$ & $2(8.0)$ & \\
\hline Previous therapies, $n(\%)$ & & & & 0.081 \\
\hline Naive & $100(37.2)$ & 95 (38.9) & $5(20.0)$ & \\
\hline Experienced & $169(62.8)$ & $149(61.1)$ & $20(80.0)$ & \\
\hline Treatment regime, $n(\%)$ & & & & 0.028 \\
\hline $3 \mathrm{D}$ & $30(11.2)$ & $27(11.1)$ & $3(12.0)$ & \\
\hline $\mathrm{SOF}+\mathrm{DCV} \pm \mathrm{RBV}$ & $83(30.9)$ & 77 (31.6) & $6(24.0)$ & \\
\hline $\mathrm{SOF}+\mathrm{LDV} \pm \mathrm{RBV}$ & $94(34.9)$ & $85(34.8)$ & $9(36.0)$ & \\
\hline $\mathrm{SOF}+\mathrm{RBV}$ & $31(11.5)$ & 29 (11.9) & $2(8.0)$ & \\
\hline $\mathrm{SOF}+\mathrm{SMV} \pm \mathrm{RBV}$ & $27(10.0)$ & $25(10.2)$ & $2(8.0)$ & \\
\hline Others & $4(1.5)$ & $1(0.4)$ & $3(12.0)$ & \\
\hline SVR12, $n(\%)$ & & & & 0.023 \\
\hline Yes & $242(90.0)$ & $222(91.0)$ & $20(80.0)$ & \\
\hline No & $17(6.3)$ & $12(4.9)$ & $5(20.0)$ & \\
\hline Lost to follow-up & $10(3.7)$ & $10(4.1)$ & $0(0.0)$ & \\
\hline Platelets/nL & $112(18-400)$ & $102(19-400)$ & $111(18-164)$ & 0.779 \\
\hline Bilirubin, mg/dL & $1.36(0.3-9.5)$ & $1.0(0.3-9.5)$ & $1.1(0.5-2.7)$ & 0.844 \\
\hline Albumin, mg/dL & $3.78(2.0-4.9)$ & $3.9(2.0-4.9)$ & $3.9(2.7-4.6)$ & 0.994 \\
\hline Creatinine, $\mathrm{mg} / \mathrm{dL}$ & $0.86(0.5-2.3)$ & $0.8(0.5-2.3)$ & $0.8(0.5-1.6)$ & 0.618 \\
\hline Prothrombin time, $\%$ & $83.5(22.0-124.0)$ & $86.0(22.0-124.0)$ & $84.0(58.0-110.0)$ & 0.797 \\
\hline GGT, U/L & $165(14-1,510)$ & $100(14-1,510)$ & $180(37-916)$ & 0.008 \\
\hline GOT, U/L & $97(17-411)$ & $83(17-411)$ & $88(31-268)$ & 0.920 \\
\hline GPT, U/L & $91(15-832)$ & $79(15-832)$ & 73 (30-195) & 0.753 \\
\hline
\end{tabular}

Variables are expressed as medians (full ranges) or as numbers (proportions), as appropriate. All $p$ values reported are two-sided. Statistical significance (in bold typeface) was defined as $p \leq 0.05$. DAA, direct-acting antiviral; HCC, hepatocellular carcinoma; MELD, Model for End-Stage Liver Disease; SOF, sofosbuvir; DCV, daclatasvir; RBV, ribavirin; LDV, ledipasvir; SMV, simeprevir; SVR12, sustained virologic response, undetectable HCV RNA 12 weeks after the end of therapy.

Overall, the predominant HCV genotype was genotype 1 (605 patients; 73.9\%), and 445 patients (54.3\%) had been treatment naïve before the start of DAA therapy. In total, $764 / 819$ patients (93.3\%) achieved SVR12, while only 32/819 (3.9\%) had virologic failure and $23(2.8 \%)$ were lost to follow-up within 12 weeks after the end of antiviral therapy. The median laboratory results for liver function were all within normal ranges at baseline (Table 1). 
Finkelmeier et al.: HCC Risk following HCV Treatment

\section{Comparison of DAA-Treated Patients with and without de novo HCC}

We compared the clinical findings of the patients with de novo HCC and those without HCC in a univariate analysis. Of the 25 patients with de novo HCC, 24 (96\%) had ChildTurcotte-Pugh (CTP) class A cirrhosis and 1 (4.0\%) had CTP class B cirrhosis. More patients with de novo HCC were IFN treatment experienced $(n=20 / 25 ; 80.0 \%)$ as compared to the patients who did not develop HCC $(n=354 / 794 ; 44.6 \%)(p=0.001)$.

Among the patients who developed HCC, significantly more patients did not achieve SVR12 $(n=5 / 25 ; 20.0 \%)$ as compared to the non-HCC group $(n=27 / 794 ; 3.4 \%)(p=0.003)$. The patients with de novo HCC had more advanced liver disease and higher GOT and GGT baseline levels than all the other patients (Table 1).

To determine risk factors for HCC development among the cirrhotic patients, subgroups of cirrhotic patients with and without HCC were analyzed in univariate analysis. The SVR12 rate remained significantly associated with HCC development: 5 nonresponders $(20.0 \%)$ developed HCC compared to 12 (4.9\%) cirrhotic patients without SVR12 who did not develop HCC ( $p=0.023)$. Of the baseline laboratory parameters, only GGT was significantly higher in the HCC group at a median of $180 \mathrm{U} / \mathrm{L}(37-916)$ compared to $100 \mathrm{U} / \mathrm{L}(14-1,510)$ among the patients who did not develop HCC ( $p=0.008)$ (Table 2$)$.

Patient Characteristics with de novo HCC

All 25 cirrhotic patients who were diagnosed with HCC during follow-up had undergone HCC surveillance every 6 months according to guideline recommendations [17]. The median time from the end of antiviral treatment to HCC diagnosis was 312 days (0-888). Five patients were diagnosed with HCC within the first 3 months after receiving antiviral therapy. Overall, 17 patients (68\%) were diagnosed with HCC at BCLC stage A, 6 patients (24\%) at BCLC stage $\mathrm{B}$, and 2 patients (8\%) at BCLC stage C. All 17 patients with early-stage HCC underwent potentially curative treatment (microwave ablation, radiofrequency ablation, surgical resection, or liver transplantation). The remaining 8 patients were treated with transarterial chemoembolization or systemic therapy with the tyrosine kinase inhibitor sorafenib (Table 3).

\section{Factors Associated with HCC Development}

For the evaluation of risk factors that were associated with the development of de novo HCC, a Cox regression analysis was performed. In our DAA-treated cohort, de novo HCC had developed exclusively in patients with cirrhosis. Therefore, only cirrhotic patients were included in the analysis. We analyzed the dichotomous variables gender, age $>65$ years, previous treatment experience, SVR, and liver enzyme elevation (GOT, GPT, and GGT). Failure to achieve SVR was a strong risk factor for de novo HCC in the uni- and multivariate analyses (Table 4a; Fig. 2).

\section{Historical Cohort Treated with IFN and an RBV Regimen}

To evaluate the impact of failed SVR as a risk factor for HCC, we also conducted a retrospective analysis of a historical cohort of 351 patients with chronic HCV infection who had undergone IFN-based therapy for HCV infection. The median follow-up was 40 months (range 0-155).

A total of 283 patients with HCV and without cirrhosis underwent therapy with IFN/ peginterferon plus RBV (peginterferon alfa-2a/-2b monotherapy or combined with RBV), while 68 patients $(19.4 \%)$ already had established cirrhosis $(n=62$ [91.2\%] at Child-Pugh class A and $n=6[8.8 \%]$ at Child-Pugh class B). In the IFN-treated cohort, 19 patients (5.4\% or $3.6 \mathrm{HCCs} / 100$ person-years) were diagnosed with de novo HCC during a median followup of 52 months (0-116). Eleven of the 283 patients (3.9\%) had no cirrhosis when treatment was started, while $8 / 68$ patients $(11.8 \%)$ already had established cirrhosis. The patients 


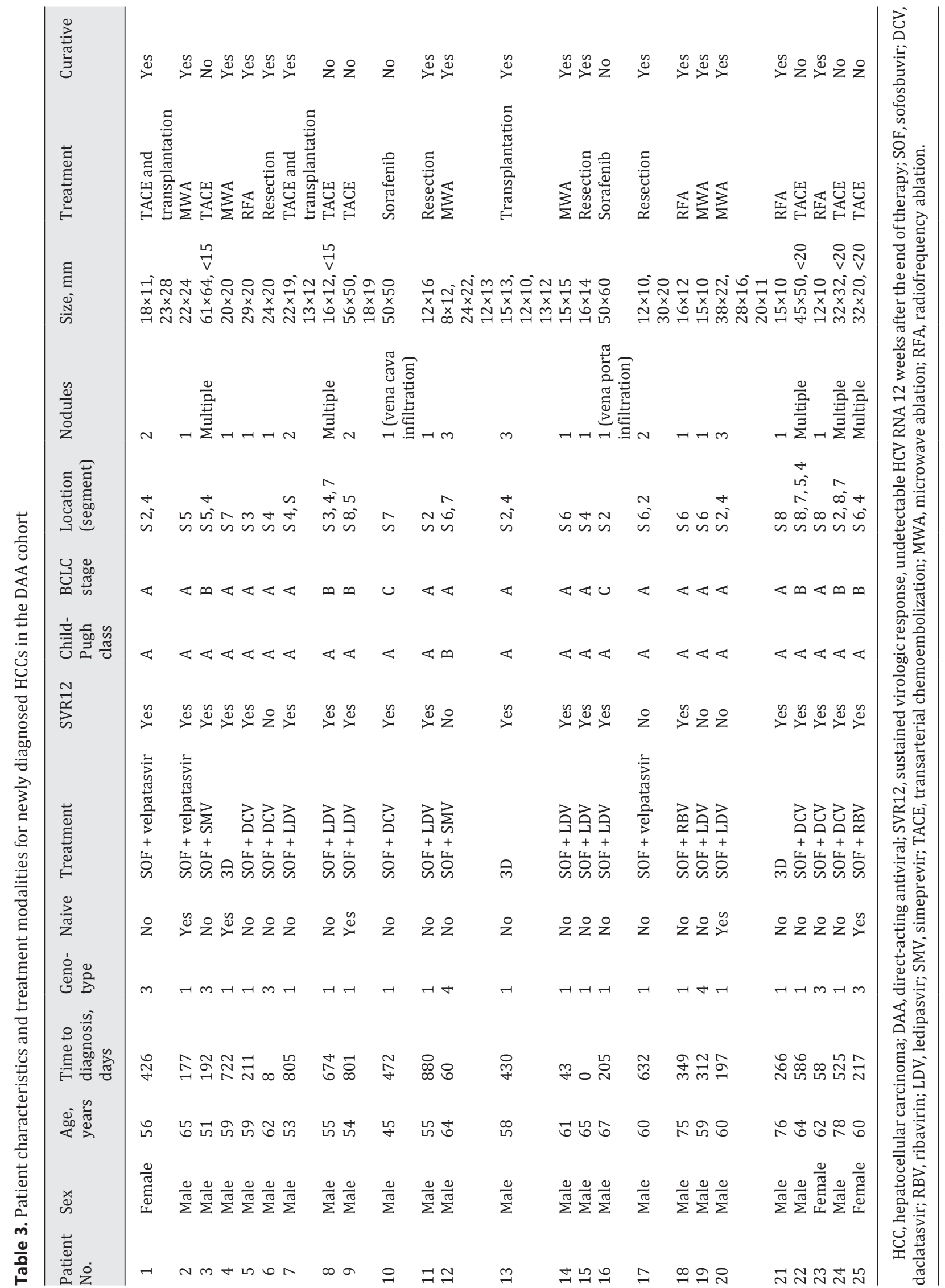




\section{Liver
Cancer}

\begin{tabular}{l}
\hline \multicolumn{2}{l|}{\begin{tabular}{l}
\hline Liver Cancer \\
\hline DOI: 10.1159/000486812
\end{tabular}} & $\begin{array}{l}\text { ○ 2018 S. Karger AG, Basel } \\
\text { www.karger.com/lic }\end{array}$ \\
\hline
\end{tabular}

Finkelmeier et al.: HCC Risk following HCV Treatment

Table 4. Univariate and multivariate analysis factors

\begin{tabular}{|c|c|c|c|c|c|c|}
\hline \multirow[b]{2}{*}{ Parameter } & \multicolumn{3}{|c|}{ Univariate analysis } & \multicolumn{3}{|c|}{ Multivariate analysis } \\
\hline & HR & $95 \% \mathrm{CI}$ & $p$ value & HR & $95 \% \mathrm{CI}$ & $p$ value \\
\hline \multicolumn{7}{|c|}{ a. Factors associated with de novo HCC in cirrhotic patients treated with DAAs } \\
\hline Male gender & 2.596 & $0.77-8.79$ & 0.125 & & & \\
\hline Age $>65$ years & 1.031 & $0.41-2.58$ & 0.948 & & & \\
\hline Treatment naïve & 0.703 & $0.26-1.91$ & 0.489 & & & \\
\hline No sustained virological response & 4.839 & $1.78-13.16$ & 0.002 & 4.448 & $1.51-13.12$ & 0.007 \\
\hline GGT > upper limit of normal & 1.655 & $0.57-4.83$ & 0.357 & & & \\
\hline GOT > upper limit of normal & 1.357 & $0.57-3.26$ & 0.459 & & & \\
\hline GPT $>$ upper limit of normal & 1.864 & $0.83-4.18$ & 0.130 & & & \\
\hline \multicolumn{7}{|c|}{ b. Factors associated with HCC in patients treated with an interferon regimen } \\
\hline Male gender & 1.292 & $0.51-3.29$ & 0.590 & & & \\
\hline Age $>65$ years & 2.222 & $0.65-7.65$ & 0.205 & & & \\
\hline No sustained virological response & 5.579 & $1.28-24.35$ & 0.022 & 4.610 & $1.02-20.76$ & 0.046 \\
\hline GGT > upper limit of normal & 1.868 & $0.76-4.62$ & 0.176 & & & \\
\hline GOT > upper limit of normal & 3.018 & $1.22-7.44$ & 0.16 & & & \\
\hline GPT $>$ upper limit of normal & 3.055 & $1.240-7.53$ & 0.15 & & & \\
\hline \multicolumn{7}{|c|}{ c. Factors associated with HCC in all patients treated with an interferon regimen and DAAs } \\
\hline Male gender & 2.487 & $1.23-5.04$ & 0.011 & 2.107 & $1.03-4.31$ & 0.041 \\
\hline Age $>65$ years & 1.804 & $0.86-3.78$ & 0.118 & & & \\
\hline No sustained virological response & 3.928 & $2.12-7.54$ & $<0.001$ & 4.348 & $1.93-9.79$ & $<0.001$ \\
\hline Cirrhosis & 7.767 & $3.91-15.42$ & $<0.001$ & 5.592 & $2.78-11.27$ & $<0.001$ \\
\hline Treatment with DAAs & 0.174 & $0.07-0.43$ & $<0.001$ & & & \\
\hline GGT > upper limit of normal & 3.005 & $1.57-5.77$ & 0.001 & & & \\
\hline GOT > upper limit of normal & 3.204 & $1.73-5.95$ & $<0.001$ & & & \\
\hline GPT > upper limit of normal & 2.023 & $1.10-3.74$ & 0.024 & & & \\
\hline
\end{tabular}

Bold typeface denotes significance. HCC, hepatocellular carcinoma; DAA, direct-acting antiviral.

with SVR24 who had no established cirrhosis did not develop HCC during follow-up (Fig. 3; Table 5).

In the IFN-treated cohort, $171 / 351$ patients (48.7\%) achieved SVR24, while only $2 / 19$ patients $(10.5 \%)$ with de novo HCC were successfully cured $(p<0.001)$. Furthermore, a higher proportion had established cirrhosis $(n=8 ; 42.1 \% ; p=0.017)$ at the beginning. Patients with de novo HCC had significantly higher levels of transaminases and lower platelet levels.

For the evaluation of risk factors that were associated with the development of de novo HCC, a Cox regression analysis was performed. In the univariate analysis of the IFN-treated cohort, failed SVR (HR 5.579; 95\% CI 1.28-24.35; $p=0.022$ ) and transaminases above the upper limit of normal increased the risk for HCC. In the multivariate analysis, failed SVR (HR 4.610; 95\% CI 1.02-20.76; $p=0.032$ ) remained an independent risk factor for de novo HCC (Table 4b).

Risk Factors for de novo HCC in the DAA-Treated and IFN-Treated Combined Cohort

To evaluate if DAA treatment is a possible risk factor for de novo HCC development, both cohorts were combined. A total of 1,170 patients were included (819 DAA treated and 351 IFN treated). De novo HCC was diagnosed in 45 patients. The univariate analysis revealed that male gender, failed SVR, presence of cirrhosis, and liver enzymes above the upper limit of normal increased the risk for de novo HCC. Treatment with DAAs was no risk factor. In the multivariate approach, male gender, failed SVR, and cirrhosis were independent risk factors for HCC development (Table 4c). 


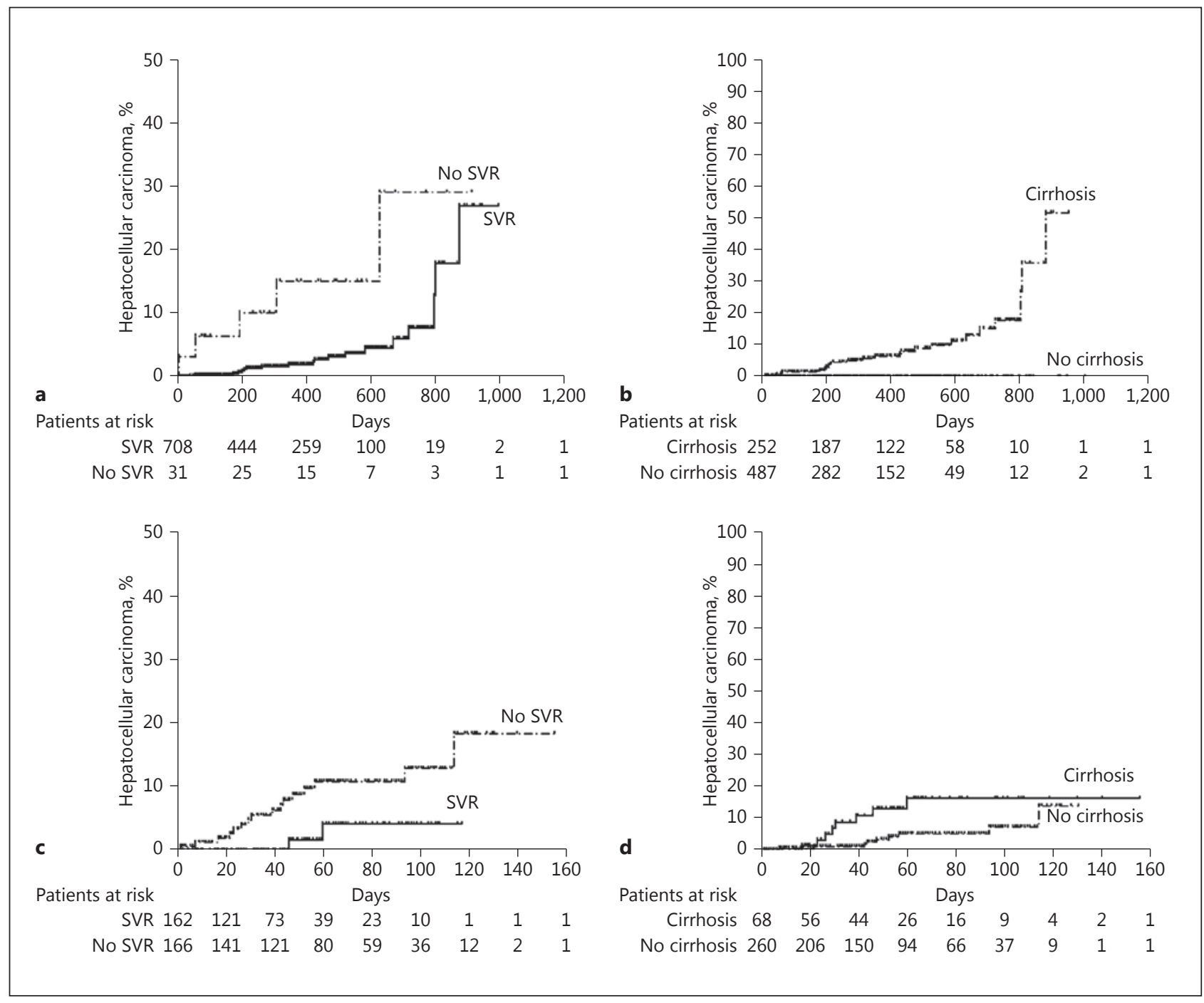

Fig. 2. a Cumulative incidence of hepatocellular carcinoma stratified according to response to treatment in the whole direct-acting antiviral (DAA) cohort ( $p<0.001$, by log-rank test). $\mathbf{b}$ Cumulative incidence of hepatocellular carcinoma stratified according to cirrhosis in the whole DAA cohort ( $p<0.001$, by log-rank test). c Cumulative incidence of hepatocellular carcinoma stratified according to response to treatment in the whole interferon (IFN) cohort ( $p=0.008$, by log-rank test). $\mathbf{d}$ Cumulative incidence of hepatocellular carcinoma stratified according to cirrhosis in the whole IFN cohort ( $p=0.001$, by log-rank test). SVR, sustained virologic response.

\section{Discussion}

HCC is one of the main complications of chronic HCV infection, with cirrhosis as its strongest risk factor. Therefore, the rationale for eradicating the virus and thereby reducing the risk for HCC development is evident. Several studies have shown a positive effect of IFNbased therapies over the last two decades; however, an SVR did not eliminate the HCC risk completely [22]. Especially the grade of fibrosis or cirrhosis is of utmost importance. Current therapies with DAAs are associated with high rates of SVR, generally exceeding $90 \%$ even among patients with cirrhosis or prior treatment failure [23, 24]. Therefore, one of the most interesting current questions concerns the impact of DAAs on HCC incidence. 
Finkelmeier et al.: HCC Risk following HCV Treatment

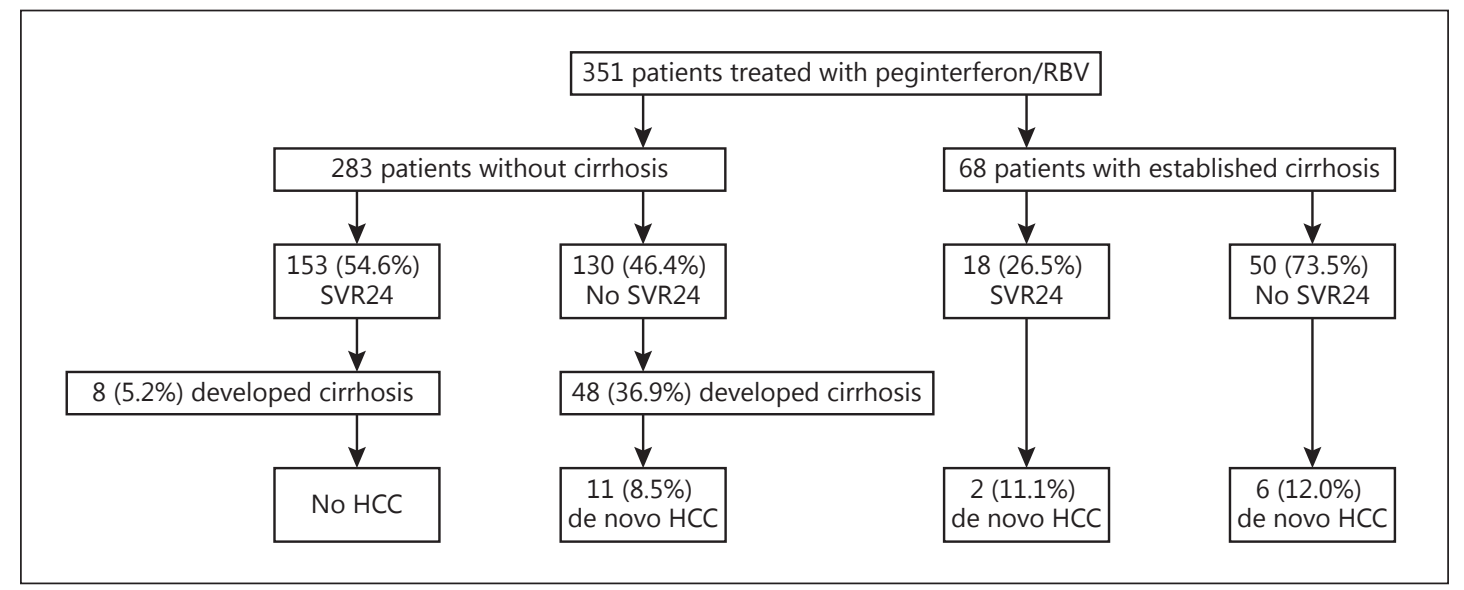

Fig. 3. Flowchart of included and excluded patients treated with interferon-based therapies and numbers of patients with hepatocellular carcinoma (HCC). SVR24, sustained virologic response, undetectable HCV RNA 24 weeks after the end of therapy.

Table 5. Patient characteristics of the IFN-treated patients

\begin{tabular}{|c|c|c|c|c|}
\hline Parameter & All patients & $\begin{array}{l}\text { No } \\
\text { diagnosed HCC }\end{array}$ & $\begin{array}{l}\text { Newly } \\
\text { diagnosed HCC }\end{array}$ & $p$ value \\
\hline \multicolumn{5}{|l|}{ Epidemiology } \\
\hline Patients, $n(\%)$ & 351 & $332(94.6)$ & $19(5.4)$ & - \\
\hline Gender (m/f), $n(\%)$ & $214(61.0) / 137(39.0)$ & $200(60.2) / 132(39.8)$ & $14(73.7) / 5(26.3)$ & 0.335 \\
\hline Age, years & $49(20-74)$ & $49(20-74)$ & $52.5(43-73)$ & 0.012 \\
\hline Follow-up, months & $46(0-155)$ & $44(0-155)$ & $52(0-116)$ & 0.408 \\
\hline Cirrhosis, $n(\%)$ & $68(19.4)$ & $60(18.1)$ & $8(42.1)$ & $\mathbf{0 . 0 1 7}$ \\
\hline Child-Pugh class, $n(\%)$ & & & & 0.653 \\
\hline A & $62(17.7)$ & $54(16.3)$ & $8(42.1)$ & \\
\hline B & $6(1.7)$ & $6(1.8)$ & $0(0.0)$ & \\
\hline $\mathrm{C}$ & $0(0.0)$ & $0(0.0)$ & $0(0.0)$ & \\
\hline Genotype, $n(\%)$ & & & & 0.706 \\
\hline 1 & $259(73.8)$ & $243(73.2)$ & $16(84.2)$ & \\
\hline 2 & $16(4.6)$ & $16(4.8)$ & $0(0.0)$ & \\
\hline 3 & $62(17.7)$ & $61(4.8)$ & $1(5.3)$ & \\
\hline 4 & $14(4.0)$ & $12(3.6)$ & $2(10.5)$ & \\
\hline SVR24, $n(\%)$ & & & & 0.001 \\
\hline Yes & $171(48.7)$ & $169(50.9)$ & $2(10.5)$ & \\
\hline No & $180(51.3)$ & $163(49.1)$ & $17(89.5)$ & \\
\hline Platelets/nL & $186(33-469)$ & $190(33-469)$ & $88(33-249)$ & $<0.001$ \\
\hline Bilirubin, mg/dL & $0.6(0.1-9.0)$ & $0.6(0.1-4.4)$ & $0.95(0.4-9.0)$ & $<0.001$ \\
\hline Creatinine, $\mathrm{mg} / \mathrm{dL}$ & $0.83(0.3-2.1)$ & $0.83(0.3-2.07)$ & $0.81(0.5-1.03)$ & 0.176 \\
\hline GGT, U/L & $32(3-487)$ & $31(3-487)$ & $118(29-356)$ & $<0.001$ \\
\hline GOT, U/L & $28(5-299)$ & $27(5-299)$ & $102(11-176)$ & $<0.001$ \\
\hline GPT, U/L & $29(3-349)$ & $28(3-349)$ & $105(17-186)$ & $<0.001$ \\
\hline
\end{tabular}

Variables are expressed as medians (full ranges) or as numbers (proportions), as appropriate. All $p$ values reported are two-sided. Statistical significance (in bold typeface) was defined as $p \leq 0.05$. IFN, interferon; HCC, hepatocellular carcinoma; SVR24, sustained virologic response, undetectable HCV RNA 24 weeks after the end of therapy. 
Finkelmeier et al.: HCC Risk following HCV Treatment

Of IFN-based therapies, which were mostly administered to noncirrhotic patients, it is known that achieving an SVR decreased the risk for de novo HCC by up to $80 \%$ among patients with fibrosis [25, 26]. However, some studies from the era of IFN-based therapies found a relevant short- and long-term risk for HCC development among patients with cirrhosis who were successfully treated [27-29].

In a Japanese study the cumulative HCC rates after successful IFN therapy were 3.1, 10.1, and $15.9 \%$ at 5,10 , and 15 years, respectively, compared with $15.8,35.5$, and $42.3 \%$ among patients without an SVR. One important risk factor in that study was a fibrosis stage $>2$ [30]. In another Japanese study, the cumulative incidence of HCC among more than 1,000 patients with an SVR after IFN therapy was 3\% a median follow-up time of 37 months after treatment [31]; again, advanced fibrosis ( $\mathrm{F}>3$ ) was a significant risk factor for HCC development. Of note, HCC development may occur in patients without or with only mild fibrosis $(\mathrm{F}<2)$. In Japanese trials, HCC risks of about 1-2\% among patients with F1-2 fibrosis were described [32]. In contrast, little is known about the HCC risk in Europeans with HCV infection. Only a few cases of HCC are described among patients with fibrosis stages of $0-2$ up to 10 years after an SVR $[33,34]$.

Most of these studies are of a retrospective nature with preselected patients and show a high degree of heterogeneity, making it difficult to assess the actual level of benefit obtained by IFN treatment $[35,36]$.

In our retrospective cohort of IFN-treated patients, 5.4\% of all patients developed HCC. In the patients with SVR24 and without cirrhosis at the start of treatment, no HCC was diagnosed during the long-term follow-up (although cirrhosis development was observed in a subset of these patients), while at least $8.5 \%$ of all patients without cirrhosis but with treatment failure developed cirrhosis and HCC. Only looking at those patients with already established cirrhosis, the rate was higher, with $11.8 \%$ of the patients developing de novo HCC.

Interestingly, in our DAA cohort, treatment failure was the most important risk factor for de novo HCC. Moreover, all patients who developed HCC had cirrhosis. This stresses the need for early treatment interventions before cirrhosis is established. Our data agree with epidemiological data describing that HCC in HCV-infected patients develops with established cirrhosis in $2-7 \%$ of patients annually [37].

In 2016, two studies reported higher rates of HCC recurrence after DAA-induced SVR [15, 16]. However, several other studies and an extensive meta-analysis could not replicate these findings [38]. In part, the different initial treatment modalities (resection/ablation vs. chemoembolization) and follow-up durations probably explain the varying findings [39, 40].

Only few data are currently available on de novo HCC development in patients treated with DAAs. Conti et al. [15] analyzed 344 cirrhotic patients and found de novo HCC in 9 of 285 patients (3.16\%). In a risk factor analysis, they found that CTP stage B, more severe liver fibrosis, lower platelet count, and previous HCC were significantly associated with HCC. However, this study also included patients with a previous history of HCC.

Cheung et al. [41] prospectively collected data on 408 patients with decompensated cirrhosis. Seventeen patients (5.4\%) developed HCC over a period of 15 months (15 with de novo and 2 with recurrent HCC). There was a reduced rate of new HCC diagnoses during a follow-up period of 15 months among patients with SVR24 compared to those without SVR24 (5.4 vs. $11.2 \%$ ). Kanwal et al. [42] recently analyzed 22,500 patients treated with DAAs with an SVR12 rate of $86.7 \%$. Thirty-nine percent of the patients had established cirrhosis and 271 HCCs (1.2\%) were detected. They found a 76\% reduction in HCC risk for patients with an SVR versus those with no SVR: the incidence rate among nonresponders was 3.45 versus 0.9 HCCs/100 person-years among responders. Patients with cirrhosis had the highest annual incidence rate of HCC. Toyoda et al. [43] reported that the annual incidence of HCC after an SVR among patients receiving an IFN-free therapy may be more than 2 -fold higher than that 
Finkelmeier et al.: HCC Risk following HCV Treatment

among patients achieving an SVR via an IFN-based therapy. More recently, Ioannou et al. [44] published data from the United States Veterans database of HCV-infected patients treated with either IFN and/or DAAs. In this study, de novo HCC was observed in 1-3/100 personyears across the different cohorts and, consistent with our findings, the authors reported a significant decrease in HCC risk following an SVR. Finally, Mettke et al. [45] investigated patients with cirrhosis and DAA treatment compared to untreated cirrhotic patients and could not find any difference in the short-term risk of HCC development regarding DAA treatment as well.

Taken together, the incidence rates of de novo HCC after DAA treatment vary between 1 and $9 \%$, most likely due to differing baseline characteristics of the patient cohorts. We found an overall incidence rate of $3.1 \%$ (3.6 HCCs/100 person-years) in the entire DAA-treated cohort. However, when analyzing only the cirrhotic patients, the rate was $9.3 \%$ (8.9 HCCs $/ 100$ person-years). In our cohort, 5 patients were diagnosed with HCC during and not after DAA therapy, suggesting this was a growth from cancers which had been radiologically undetectable at the beginning of DAA therapy rather than very early de novo development. Most of the tumors were very small at diagnosis. This underlines the recent improvement in imaging modalities that helps in improving detection rates and overall HCC surveillance. We found no short-term risk among the patients with advanced fibrosis (F3), and this underlines the finding that established cirrhosis increases the HCC risk.

It is difficult to compare our DAA-treated patients to those who received IFN in the historical cohorts because the demographic data were different. Nonetheless, the HCC rates seem to be comparable between the different cohorts. In recent discussions and conference proceedings, several factors that may influence the higher detection rate of HCCs in DAAtreated patients were brought up. Importantly, the average follow-up was shorter and the average age was higher in DAA studies than in IFN studies [38]. In a large Scottish database study, cirrhotic patients with DAA treatment compared to IFN-treated patients were older and had lower platelet counts and more often CTP stages B and C. Moreover, significantly more DAA-treated patients were IFN experienced [46]. This compares well to our findings and proves that time clearly has left its mark, with DAA-treated patients being older and having more advanced disease. Moreover, our data clearly imply that in patients with compensated cirrhosis, eradication of HCV should be achieved before liver function is impaired.

According to recent guideline recommendations, surveillance of HCC with liver imaging should be pursued twice annually for an indefinite duration in all patients with stage 3 fibrosis or established cirrhosis after HCV eradication, but surveillance of HCC is not recommended for patients with stage $0-2$ fibrosis after an SVR. An intensification of the HCC screening frequency in the immediate post-SVR context is not currently recommended [47]. As patients with failed SVRs are at very high risk, we suggest that these patients should be under very close surveillance (e.g., ultrasound surveillance every 3 months) even during antiviral therapy, and DAA rescue therapies should be evaluated.

The value of DAA therapy for patients with cirrhosis in reducing the risk of HCC development will remain under debate until more long-term data are available. Our data after 12 months of follow-up suggest that in patients with cirrhosis there is no short-term reduction in HCC risk; however, the risk was not increased when compared to IFN-treated patients either.

\section{Disclosure Statement}

F.F., K.-H.P., and F.K. report no conflict of interest. G.D. reports personal fees from AbbVie. B.K. reports personal fees from AbbVie, Falk, and Bristol-Myers Squibb. S.Z. reports personal fees as a consultant and/or speaker for AbbVie, Bristol-Myers Squibb, Falk, Gilead, Janssen, and Merck/MSD. C.S. reports personal fees as a consultant and/or speaker for Abbott, AbbVie, Bristol-Myers Squibb, Gilead, Intercept, Janssen, Merck/ 
MSD, Roche, and Siemens, as well as research grants from Abbott, Gilead, Merck/MSD, and Janssen. J.V. reports personal fees from Abbott, AbbVie, Bristol-Myers Squibb, Gilead, MSD, Medtronic, and Roche outside the submitted work. O.W. received travel grants from AbbVie, Bayer, and Gilead. He reports personal fees from Bayer and Bristol-Myers Squibb.

\section{Author Contributions}

F.F., J.V., and O.W. performed the research. F.F., G.D., B.K., K.-H.P., F.K., J.V., and O.W. collected and analyzed the data. F.F., J.V., and O.W. designed the research study and wrote the paper. S.Z., C.S., J.V., and O.W. contributed to the design of the study. All authors approved the final version of the manuscript.

\section{References}

1 Lavanchy D: Evolving epidemiology of hepatitis C virus. Clin Microbiol Infect 2011;17:107-115.

-2 Polaris Observatory HCV Collaborators S, Zeuzem S, Manns M, et al: Global prevalence and genotype distribution of hepatitis C virus infection in 2015: a modelling study. Lancet Gastroenterol Hepatol 2017;2:161176.

3 Thein HH, Yi Q, Dore GJ, Krahn MD: Estimation of stage-specific fibrosis progression rates in chronic hepatitis C virus infection: a meta-analysis and meta-regression. Hepatology 2008;48:418-431.

4 Seeff LB: Natural history of chronic hepatitis C. Hepatology 2002;36(suppl 1):S35-S46.

5 Poynard T, Mathurin P, Lai CL, et al: A comparison of fibrosis progression in chronic liver diseases. J Hepatol 2003;38:257-265.

6 El-Serag HB: Hepatocellular carcinoma. N Engl J Med 2011;365:1118-1127.

7 Fattovich G, Stroffolini T, Zagni I, Donato F: Hepatocellular carcinoma in cirrhosis: incidence and risk factors. Gastroenterology 2004;127(suppl 1):S35-S50.

-8 Lok AS, Seeff LB, Morgan TR, et al: Incidence of hepatocellular carcinoma and associated risk factors in hepatitis C-related advanced liver disease. Gastroenterology 2009;136:138-148.

-9 Sangiovanni A, Del Ninno E, Fasani P, et al: Increased survival of cirrhotic patients with a hepatocellular carcinoma detected during surveillance. Gastroenterology 2004;126:1005-1014.

10 Benvegnù L, Gios M, Boccato S, Alberti A: Natural history of compensated viral cirrhosis: a prospective study on the incidence and hierarchy of major complications. Gut 2004;53:744-749.

11 Aizawa Y, Shibamoto Y, Takagi I, Zeniya M, Toda G: Analysis of factors affecting the appearance of hepatocellular carcinoma in patients with chronic hepatitis C. A long term follow-up study after histologic diagnosis. Cancer 2000;89:53-59.

12 Khan MH, Farrell GC, Byth K, et al: Which patients with hepatitis C develop liver complications? Hepatology 2000;31:513-520.

13 Lawitz E, Poordad FF, Pang PS, et al: Sofosbuvir and ledipasvir fixed-dose combination with and without ribavirin in treatment-naive and previously treated patients with genotype 1 hepatitis $C$ virus infection (LONESTAR): an open-label, randomised, phase 2 trial. Lancet 2014;383:515-523.

14 Guarino M, Morisco F, Valvano MR, et al: Systematic review: interferon-free regimens for patients with HCVrelated Child C cirrhosis. Aliment Pharmacol Ther 2017;45:1193-1200.

15 Conti F, Buonfiglioli F, Scuteri A, et al: Early occurrence and recurrence of hepatocellular carcinoma in HCVrelated cirrhosis treated with direct-acting antivirals. J Hepatol 2016;65:727-733.

16 Reig M, Marino Z, Perello C, et al: Unexpected high rate of early tumor recurrence in patients with HCV-related HCC undergoing interferon-free therapy. J Hepatol 2016;65:719-726.

-17 European Association for the Study of the Liver; European Organisation for Research and Treatment of Cancer: EASL-EORTC clinical practice guidelines: management of hepatocellular carcinoma. J Hepatol 2012; 56:908-943.

18 Castéra L, Vergniol J, Foucher J, et al: Prospective comparison of transient elastography, Fibrotest, APRI, and liver biopsy for the assessment of fibrosis in chronic hepatitis C. Gastroenterology 2005;128:343-350.

19 Friedrich-Rust M, Ong MF, Martens S, et al: Performance of transient elastography for the staging of liver fibrosis: a meta-analysis. Gastroenterology 2008;134:960-974.

20 European Association for Study of Liver: EASL recommendations on treatment of hepatitis C 2015. J Hepatol 2015;63:199-236.

21 Sarrazin C, Berg T, Buggisch P, et al: S3 guideline hepatitis C addendum (in German). Z Gastroenterol 2015;53: 320-334.

22 Nishiguchi S, Kuroki T, Nakatani S, et al: Randomised trial of effects of interferon-alpha on incidence of hepatocellular carcinoma in chronic active hepatitis C with cirrhosis. Lancet 1995;346:1051-1055.

23 Poordad F, Hezode C, Trinh R, et al: ABT-450/r-ombitasvir and dasabuvir with ribavirin for hepatitis C with cirrhosis. N Engl J Med 2014;370:1973-1982. 
Finkelmeier et al.: HCC Risk following HCV Treatment

24 Bourlière M, Gordon SC, Flamm SL, et al: Sofosbuvir, velpatasvir, and voxilaprevir for previously treated HCV infection. N Engl J Med 2017;376:2134-2146.

-25 Shen YC, Hsu C, Cheng CC, et al: A critical evaluation of the preventive effect of antiviral therapy on the development of hepatocellular carcinoma in patients with chronic hepatitis $\mathrm{C}$ or B: a novel approach by using metaregression. Oncology 2012;82:275-289.

26 Morgan RL, Baack B, Smith BD, et al: Eradication of hepatitis C virus infection and the development of hepatocellular carcinoma: a meta-analysis of observational studies. Ann Intern Med 2013;158(pt 1):329-337.

27 Morgan TR, Ghany MG, Kim HY, et al: Outcome of sustained virological responders with histologically advanced chronic hepatitis C. Hepatology 2010;52:833-844.

28 Aleman S, Rahbin N, Weiland O, et al: A risk for hepatocellular carcinoma persists long-term after sustained virologic response in patients with hepatitis C-associated liver cirrhosis. Clin Infect Dis 2013;57:230-236.

29 van der Meer AJ, Veldt BJ, Feld JJ, et al: Association between sustained virological response and all-cause mortality among patients with chronic hepatitis $\mathrm{C}$ and advanced hepatic fibrosis. JAMA 2012;308:2584-2593.

-30 Yamashita N, Ohho A, Yamasaki A, et al: Hepatocarcinogenesis in chronic hepatitis C patients achieving a sustained virological response to interferon: significance of lifelong periodic cancer screening for improving outcomes. J Gastroenterol 2014;49:1504-1513.

31 Nagaoki Y, Aikata H, Nakano N, et al: Development of hepatocellular carcinoma in patients with hepatitis C virus infection who achieved sustained virological response following interferon therapy: a large-scale, longterm cohort study. J Gastroenterol Hepatol 2016;31:1009-1015.

-32 Ikeda M, Fujiyama S, Tanaka M, et al: Risk factors for development of hepatocellular carcinoma in patients with chronic hepatitis $C$ after sustained response to interferon. J Gastroenterol 2005;40:148-156.

-33 Sewell JL, Stick KM, Monto A: Hepatocellular carcinoma after sustained virologic response in hepatitis C patients without cirrhosis on a pretreatment liver biopsy. Eur J Gastroenterol Hepatol 2009;21:225-229.

-34 Scherzer TM, Reddy KR, Wrba F, et al: Hepatocellular carcinoma in long-term sustained virological responders following antiviral combination therapy for chronic hepatitis C. J Viral Hepat 2008;15:659-665.

-35 Craxì A, Cammà C: Prevention of hepatocellular carcinoma. Clin Liver Dis 2005;9:329-346, viii.

-36 Cardoso AC, Moucari R, Figueiredo-Mendes C, et al: Impact of peginterferon and ribavirin therapy on hepatocellular carcinoma: incidence and survival in hepatitis C patients with advanced fibrosis. J Hepatol 2010;52: 652-657.

37 Sangiovanni A, Prati GM, Fasani P, et al: The natural history of compensated cirrhosis due to hepatitis C virus: a 17-year cohort study of 214 patients. Hepatology 2006;43:1303-1310.

-38 Waziry R, Hajarizadeh B, Grebely J, et al: Hepatocellular carcinoma risk following direct-acting antiviral HCV therapy: a systematic review, meta-analyses, and meta-regression. J Hepatol 2017;67:1204-1212.

-39 Kolly P, Waidmann 0, Vermehren J, et al: Hepatocellular carcinoma recurrence after direct antiviral agent treatment: a European multicentre study. J Hepatol 2017;67:876-878.

-40 ANRS Collaborative Study Group on Hepatocellular Carcinoma (ANRS C022 HEPATHER, C012 CirVir, and CO23 CUPILT cohorts): Lack of evidence of an effect of direct-acting antivirals on the recurrence of hepatocellular carcinoma: data from three ANRS cohorts. J Hepatol 2016;65:734-740.

41 Cheung MCM, Walker AJ, Hudson BE, et al: Outcomes after successful direct-acting antiviral therapy for patients with chronic hepatitis C and decompensated cirrhosis. J Hepatol 2016;65:741-747.

$\$ 42$ Kanwal F, Kramer J, Asch SM, et al: Risk of hepatocellular cancer in HCV patients treated with direct acting antiviral agents. Gastroenterology 2017;153:996-1005.e1.

43 Toyoda H, Kumada T, Tada T: Changes in patient backgrounds may increase the incidence of HCC after SVR in the era of IFN-free therapy for HCV. Hepatology 2016;64:1818-1819.

44 Ioannou GN, Green PK, Berry K: HCV eradication induced by direct-acting antiviral agents reduces the risk of hepatocellular carcinoma. J Hepatol 2017, Epub ahead of print.

45 Mettke F, Schlevogt B, Deterding K, et al: Interferon-free therapy of chronic hepatitis C does not change the short-term risk for de novo hepatocellular carcinomas in patients with liver cirrhosis. J Hepatol 2017;66:S210S211.

-46 Innes H, Barclay ST, Hayes PC, et al: The risk of hepatocellular carcinoma in cirrhotic patients with hepatitis C and sustained viral response: role of the treatment regimen. J Hepatol 2017, Epub ahead of print.

47 Jacobson IM, Lim JK, Fried MW: American Gastroenterological Association Institute Clinical Practice Update - expert review: care of patients who have achieved a sustained virologic response after antiviral therapy for chronic hepatitis C infection. Gastroenterology 2017;152:1578-1587. 